\title{
Downregulation of LncRNA-MEG3 promotes HTR8/SVneo cells apoptosis and attenuates its migration by repressing Notch1 signal in preeclampsia
}

\author{
Rongli Wang ${ }^{1}$ and $\mathrm{Li} \mathrm{Zou}^{2}$ \\ ${ }^{1}$ Department of Obstetrics and Gynecology, Xi'an Fourth Hospital, Shanxi, China and ${ }^{2}$ Department of Obstetrics \\ and Gynecology, Union Hospital, Tongji Medical College, Huazhong University of Science and Technology, \\ Wuhan, China \\ Correspondence should be addressed to L Zou; Email: xiehezouli@hust.edu.cn
}

\begin{abstract}
A successful pregnancy crucially depends on well-regulated extravillous trophoblast migration and invasion. Maternally expressed gene 3 (MEG3) is a long noncoding RNA that plays an important role in regulating trophoblast cells cell function. As previously reported, the expression of MEG3 was reduced in preeclampsia, and downregulation of MEG3 could suppress trophoblast cells migration and promote its apoptosis. However, the downstream regulatory mechanism of MEG3 remains unknown. As reported, MEG3 could inhibit cell proliferation in endometrial carcinoma by regulating Notch signaling. Our previous studies have demonstrated that Notch1 is downregulated in preeclampsia and that inhibiting the expression of Notch1 could promote trophoblast cell apoptosis. Therefore, this study was designed to investigate the role of MEG3 and its the relationship with Notch1 in trophoblasts. In this study, the mRNA expression levels of both MEG3 and Notch1 were decreased in preeclampsia placenta $(n=15)$ compared to the normal samples $(n=15)$. Exogenous upregulation and downregulation of MEG3 in HTR8/SVneo cells were performed to investigate the role of MEG3 in cell biological behavior and its effects on Notch1 expression. The results showed that MEG3 enhancement promoted trophoblast cell migration and invasion and inhibited cell apoptosis. Downregulation of MEG3 elicited the opposite results. Associated factors, such as matrix metalloproteinases 2 (MMP2), BAX, and Bcl-2, were examined at the mRNA and protein levels. Our study demonstrated that MEG3 could regulate Notch1 expression to modulate trophoblast cell migration, invasion, and apoptosis, which may represent the molecular mechanism of poor placentation during preeclampsia.

Reproduction (2020) 160 21-29
\end{abstract}

\section{Introduction}

Preeclampsia is a leading cause of maternal-fetal morbidity and mortality worldwide (American College of Obstetricians and Gynecologists \& Task Force on Hypertension in Pregnancy 2013). It is well known that the placenta plays a vital role in preeclampsia pathogenesis. During early placentation, extravillous trophoblasts (EVTs) invade the uterine decidua and then replace the endothelial layer and to complete spiral artery remodeling, leading the uterine spiral arteries to became high-capacitance and low-resistance vessels and increasing uteroplacental perfusion (Brosens \& Renaer 1972). However, in preeclampsia, this process is interrupted (Meekins et al. 1994), EVT migration and invasion ability are decreased (de Groot et al. 1996) and apoptosis is increased (Myatt 2002), which are all detrimental to uterine spiral artery remodeling. Therefore, additional efforts on studying the altered function of trophoblast cells are of great value to deepen our understanding of the molecular mechanism of preeclampsia.
Maternally expressed gene 3 (MEG3), 1645 bp in length, is a long noncoding RNA gene that is located on human chromosome 14q32 (Balik et al. 2013). MEG3 is necessary for normal growth and development of the human body and is expressed in many normal tissues (Zhou et al. 2012), especially in the brain, ovary, and placenta (Zhang et al. 2003). Moreover, it has been demonstrated that MEG3 plays a vital role in the regulation of cell biological functions, such as migration and apoptosis (Wang et al. 2015, Sun et al. 2016). Our previous studies demonstrated that MEG3 could control vascular smooth muscle cell (VSMC) migration and apoptosis (Liu et al. 2017b, 2019). Other studies have reported that the expression of MEG3 was decreased in the placenta of preeclampsia patients at the trophoblast cell level (Zhang et al. 2015). However, a deeper understanding of the downstream molecular mechanism of MEG3 in the trophoblasts is unknown.

Previous studies have demonstrated that MEG3 could regulate cell proliferation by inhibiting the Notch signaling pathway in endometrial carcinoma (Guo et al. 
2016). Notch signaling is a highly conserved pathway that regulates intercellular communication. In particular, Notch1 has been demonstrated to be involved in the pathogenesis of preeclampsia (Qing-qing et al. 2015, Liu et al. 2016), and mouse embryos lacking Notch1 showed defects in angiogenesis (Limbourg et al. 2005). Therefore, we sought to investigate whether MEG3 and Notch1 have cooperating roles in regulating trophoblast cell migration and apoptosis, which might be important for the pathogenesis of preeclampsia.

In this study, we first detected the mRNA expression levels and the correlation between MEG3 and Notch1 in the preeclampsia placenta samples compared to controls. Second, we examined the role of MEG3 in trophoblast cell migration and apoptosis. Finally, we studied the molecular mechanism of MEG3 modulating Notch1 via gain- and loss-of-function experiments.

\section{Materials and methods}

\section{Placenta tissues}

This study was approved by the Ethics Committee of Union Hospital, Huazhong University of Science and Technology (HUST), Wuhan, China. A total of 15 paired specimens were obtained from the third-trimester placenta of preeclampsia patients and normal controls from September 2018 to February 2019. After the delivery of the placenta, the specimens around $3-5 \mathrm{~cm}$ of the umbilical cord attachment site (on the chorionic side, full-thickness) were clipped. All of the pregnant women underwent cesarean sections in the third trimester, and all participants signed an informed consent form that allowed us to perform research with the obtained tissues. Furthermore, the inclusion and exclusion criteria of the population have been described previously (Wang et al. 2019).

\section{Cell culture and treatment}

The EVT cell line HTR8/SVneo was kindly gifted by Dr Charles Graham (Queen's University, Canada). Cells were cultured in RPMI 1640 medium (HyClone, USA) that contained 10\% fetal bovine serum (FBS, Gibco - BRL - Life Technologies). The culture medium was changed every 2 days. Lipofectamine 2000 reagent (Invitrogen) was used for HTR8/SVneo transient transfection. According to the manufacturer's protocol, cells were seeded into a six-well plate. When the cells reached approximately $80 \%$ confluence, they were transfected with $4.0 \mu \mathrm{g}$ of vector DNA by $4 \mu \mathrm{L}$ Lipofectamine 2000 per well. The transfection efficiency was determined by qRT-PCR and Western blot. Overexpression of MEG3 was achieved by transfection of the pcDNA-MEG3 vector (GeneChem Co. Ltd, Shanghai, China), and an empty vector was designed as a negative control (NC group). To inhibit MEG3 and Notch1 expression, cells were transfected with DNA plasmids against MEG3 (sh-MEG3-1, sh-MEG3-2, sh-MEG3-3) and Notch1 (sh-Notch1-1, sh-Notch1-2) separately. Negative controls for sh-MEG3 (sh-MEG3-NC) and sh-Notch1 (sh-Notch1-NC) were also included.

\section{$R N A$ isolation, reverse transcription, and quantitative real-time $P C R$}

Total RNA from placental tissues and HTR8/SVneo cells was isolated using RNAiso Plus (Takara) and reverse transcribed into cDNA using Prime-Script ${ }^{\mathrm{TM}}$ RT Master Mix (Takara). PCR was performed using SYBR Premix ExTaq ${ }^{\text {TM }}$ (Takara) on the StepOne Plus ${ }^{\mathrm{TM}}$ Real-Time PCR system (Applied Biosystems, Inc.). The reaction conditions were as follows: a denaturation step $\left(95^{\circ} \mathrm{C}\right.$ for $\left.30 \mathrm{~s}\right)$, followed by 40 cycles at $95^{\circ} \mathrm{C}$ for $30 \mathrm{~s}, 60^{\circ} \mathrm{C}$ for $30 \mathrm{~s}$, and extension at $72^{\circ} \mathrm{C}$ for $60 \mathrm{~s}$ (Wang et al. 2019). The PCR primer sequences used in our study were as follows: MEG3 - F: TGCGGAAGAGGCCCTGAT, MEG3 - R: GTCCAGAGTCTCTGGGTCCA; GAPDH (internal control) - F: ACAACTTTGGTATCGTGGAAGG, GAPDH - R: GCCATCACGCCACAGTTTC; Notch1 - R: CTTGTACTCCGTCAGCGTGA; matrix metalloproteinase 2 (MMP2) - F:TACAGGATCATTGGCTACACACC; MMP2 - R: GGTCACATCGCTCCAGAC; BCl-2 - F: TTCCACGCCGAAGGACAGCG, BCl-2 - R: GGCACTTGTGGCGGCCTGAT; and BAX - F: AGTGGCAGCTGACATGTTTT, BAX - R: GGAGGAAGTCCAATGTCCAG. The comparative $\mathrm{Ct}\left(2^{-\Delta \Delta \mathrm{Ct}}\right)$ method was used to analyze target gene expression relative to that of GAPDH.

\section{Western blot analysis}

Total proteins that were extracted from specimens and cultured cells using RIPA buffer and a BCA protein assay kit (Beyotime, Shanghai, China) were used to measure the protein concentrations. Each sample containing $60 \mu \mathrm{g}$ of protein was mixed with loading buffer, loaded onto $10 \%$ SDS-PAGE gels, and transferred onto PVDF membranes. These membranes were blocked with $0.1 \%$ TBST containing 5\% nonfat dry milk and incubated with specific primary antibodies against Notch1 (1:800, product number 65297; Abcam), MMP2 (1:1,000, product number 40994; CST, Boston, MA), Bcl-2 (1:800, product number 6139; Affinity, Cincinnati, OH), and $\operatorname{BAX}(1: 800$, product number 32503; Abcam) overnight. $\beta$-actin (1:800, product number 7018; Affinity) was used as an internal control. Then, the membranes were washed with TBST three times and incubated with peroxidase-conjugated secondary antibodies for $1 \mathrm{~h}$ at room temperature. Eventually, an enhanced chemiluminescence detection system was used to measure the density of the bands.

\section{Migration and invasion assays of HTR8/SVneo cells}

A total of $5 \times 10^{4}$ transfected HTR8/SVneo cells were resuspended in $300 \mu \mathrm{L}$ serum-free medium and seeded into the upper chamber of a 24-well transwell insert (pore size 8 $\mu \mathrm{m})$. Then $600 \mu \mathrm{L}$ complete culture medium was added into the lower chamber. After cells were incubated for $24 \mathrm{~h}$ at $37^{\circ} \mathrm{C}$, in $5 \% \mathrm{CO}_{2}$, we fixed the insert in $4 \%$ paraformaldehyde and stained it with $0.2 \%$ crystal violet. The cells that could not migrate to the lower chamber were removed using a cottontipped swab. The migrated cells were imaged and counted under an inverted phase-contrast microscope (magnification 200x, Olympus). For each insert, we counted cells in five randomly selected fields. The invasion assay was performed 
Table 1 Clinical characteristics of normal and preeclamptic pregnancies.

\begin{tabular}{lccc}
\hline Characteristics & Normal $(n=15)$ & Preeclampsia $(n=15)$ & $\boldsymbol{P}$-value \\
\hline Maternal age (year) & $30.5 \pm 0.57$ & $29.34 \pm 1.03$ & $>0.05$ \\
Maternal weight (kg) & $66.1 \pm 1.85$ & $68.8 \pm 1.36$ & $>0.05$ \\
Gestational week at delivery & $38.5 \pm 0.36$ & $36 \pm 0.67$ & $>0.05$ \\
SBP $(\mathrm{mmHg})$ & $113.55 \pm 2.36$ & $163.59 \pm 5.79$ & $<0.05$ \\
DBP $(\mathrm{mmHg})$ & $72.64 \pm 1.88$ & $108.46 \pm 3.26$ & $<0.05$ \\
Proteinuria (g/day) & $0(0 / 15)$ & $15(15 / 15)$ & $<0.05$ \\
Birth weight (g) & $3120 \pm 62.20$ & $2643 \pm 45.60$ & $<0.05$ \\
\hline
\end{tabular}

Data is presented as mean \pm S.E.M. or percentage (number/total); $P$-value $<0.05$ was considered as significant difference.

DBP: diastolic blood pressure; SBP: systolic blood pressure.

in a similar way, except that the membranes were precoated with Matrigel Mix (BD Science, Sparks, MD), the density of the cells was $8 \times 10^{4}$ per well, and the invasion time was extended to $48 \mathrm{~h}$.

\section{Flow cytometric analysis}

Transfected HTR8/SVneo cells were plated in six-well plates and incubated for $48 \mathrm{~h}$ at $37^{\circ} \mathrm{C}$ in $5 \% \mathrm{CO}_{2}$. Then, the cells were harvested and washed with cold PBS. The cells were resuspended in $200 \mu \mathrm{L}$ binding buffer, and $5 \mu \mathrm{L}$ annexin $\mathrm{V}$-fluorescein isothiocyanate and $5 \mu \mathrm{L}$ propidium iodide (PI) were added to the cells at room temperature in darkness for $10 \mathrm{~min}$. Finally, $300 \mu \mathrm{L}$ binding buffer was added to each tube, and the stained cells were analyzed using flow cytometer (FC500, MCL, Beckman, CA).

\section{Statistical analyses}

The results were presented as the mean \pm S.D., and every experiment was performed at least three times. Student's $t$-test and one-way ANOVA, followed by Newman-Keuls multiple comparison test and two-way ANOVA were used to analyze the data, using the Graphpad Prism 5 software. $P<0.05$ was considered to be statistically significant.

\section{Results}

\section{MEG3 and Notch1 expression is decreased in preeclamptic placental tissues}

The clinical characteristics of the subjects are summarized in Table 1. Paraffin-embedded placental tissues collected from preeclampsia patients and normal controls were prepared and analyzed by real-time PCR to detect the mRNA expression levels of MEG3 and Notch1. The results showed that the mRNA expression levels of MEG3 (Normal: Pre-eclampsia: 8.655 \pm 3.012 vs $0.8165 \pm 0.4050, P<0.01$ ) and Notch1 (Normal: Pre-eclampsia: $1.948 \pm 0.3920$ vs $0.6429 \pm 0.02833$, $P<0.001)$ were both decreased significantly in the preeclampsia placenta samples compared to the normal control samples (Fig. 1A). In the preeclampsia placenta, there was a positive correlation between MEG3 and Notch1 (Pearson $r=0.9117,95 \% \mathrm{Cl}(0.7381,0.9721)$, $P<0.001$, Mann-Whitney $U$ test) (Fig. 1B).

\section{MEG3 facilitated the expression of Notch1 in HTR8/ SVneo cell line}

To explore the effects of MEG3 on Notch1 expression in HTR8/SVneo cells, HTR8/SVneo cells were transfected with MEG3 and showed significantly upregulated expression of Notch1 compared to that in cells transfected with the empty vector (NC:MEG3: mRNA: $0.9900 \pm 0.005773$ vs $14.37 \pm 3.426$; protein: $1.137 \pm 0.06888$ vs $4.304 \pm 0.03355$ ) (Fig. $2 A, B, C$ and D). On the other hand, transfecting sh-MEG3 in HTR8/SVneo cell resulted in decreased mRNA and protein expression levels of Notch1 compared with transfection with sh-MEG3-NC (sh-MEG3 vs sh-MEG3-1, sh-MEG3-2, sh-MEG3-3:mRNA: $0.9885 \pm 0.00563$ vs $0.05159 \pm 0.02120 ; 0.1997 \pm 0.07406 ; 0.3283 \pm 0.1569$. protein: $\quad 0.8400 \pm 0.09238$ vs $0.2475 \pm 0.02685$; $0.1820 \pm 0.005774 ; 0.1315 \pm 0.007217)($ Fig. $2 \mathrm{E}, \mathrm{F}, \mathrm{G}$ and $\mathrm{H})$. These results demonstrated that MEG3 could facilitate Notch1 expression at the transcriptional and posttranscriptional levels in HTR8/SVneo cells.

\section{Knockdown of MEG3 inhibits HTR8/SVneo cell migration and invasion}

We further investigated the role of MEG3 knockdown on HTR8/SVneo cell migration and invasion. In the transwell
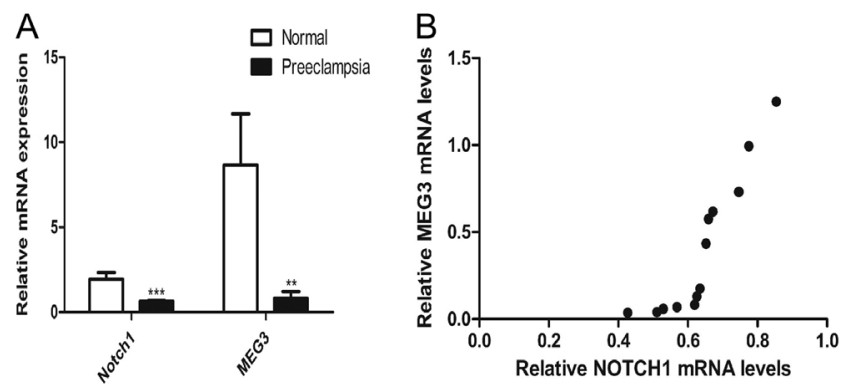

Figure 1 MEG3 and Notch1 expression in different placenta samples groups. (A) RT-PCR analysis revealed that the normal placenta produced significantly higher transcriptional levels of MEG3 and Notch 1 than the preeclampsia placenta $\left({ }^{*} P<0.05\right)$. (B) There was a positive correlation between MEG3 and Notch1 mRNA levels in preeclampsia placental tissues. (Pearson $r=0.9117,95 \% \mathrm{Cl}(0.7381$, $0.9721), * P<0.001$, Mann-Whitney $U$ test). 

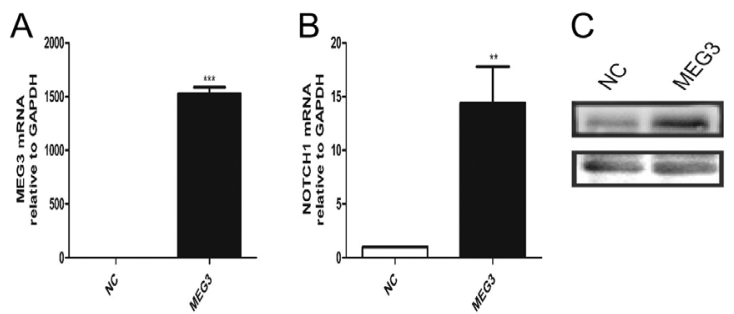

Notch1 GAPDH
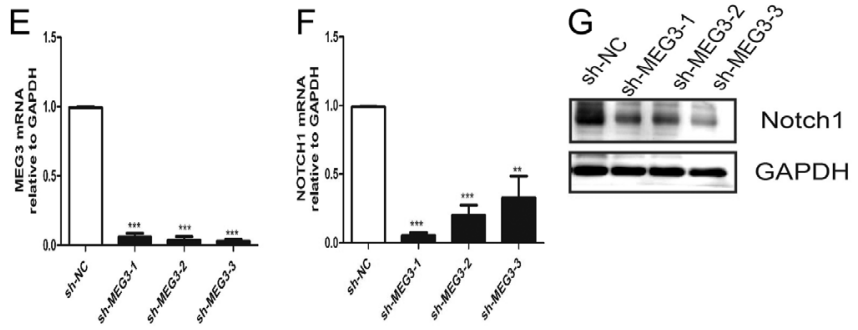

assay, MEG3 knockdown decreased HTR8/SVneo cell migration capabilities compared to the cells that were transfected with sh-MEG3-NC (sh-MEG3 vs sh-MEG3-1, sh-MEG3-2, sh-MEG3-3: 453.3 10.33 vs $165.5 \pm 18.75$ vs $226.0 \pm 13.77$ vs $145.0 \pm 13.27$ ) (Fig. $3 \mathrm{~A}$ and C). Matrigel invasion assays also demonstrated that HTR8/

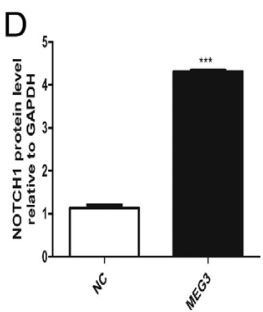

Figure 2 MEG3 facilitated the expression of Notch1 in HTR8/SVneo cell lines. qRT-PCR (A and $\mathrm{B})$ and Western blot (C and $\mathrm{D})$ indicated that stable transfection of MEG3 into HTR8/ SVneo cells resulted in upregulation of Notch1 at both the transcriptional and protein levels compared to those transfected with empty vector (NC). RT-PCR (E and F) and Western blot $(\mathrm{G}$ and $\mathrm{H})$ indicated that stable transfection of sh-MEG3 into HTR8/SVneo cells resulted in downregulation of Notch1 compared with sh-MEG3-NC-transfected cells. $\left({ }^{* *} P<0.01,{ }^{* * *} P<0.001\right)$.

SVneo cells transfected with sh-MEG3 significantly inhibited the cell invasion capability compared with sh-MEG3-NC-transfected cells (sh-MEG3 vs sh-MEG3-1, sh-MEG3-2, sh-MEG3-3: $399.7 \pm 1.202$ vs $254.4 \pm 21.89$ vs $210.5 \pm 15.68$ vs $209.2 \pm 20.98$ ) (Fig. $3 B$ and D). Furthermore, we tested the expression level of MMP2,

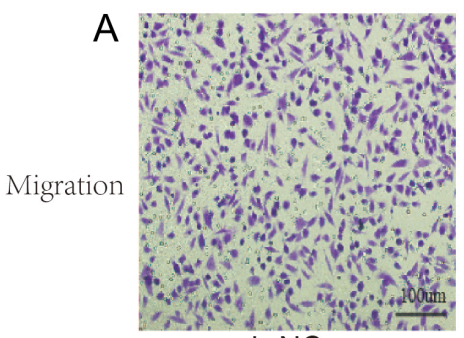

sh-NC

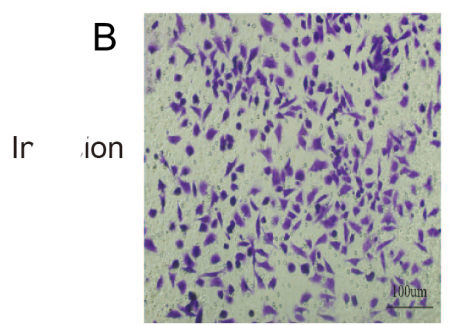

sh-NC

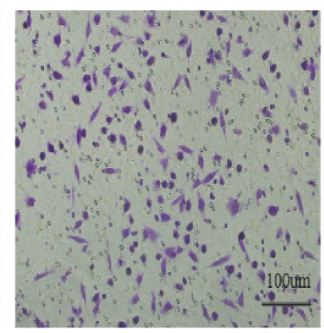

sh-MEG3-1

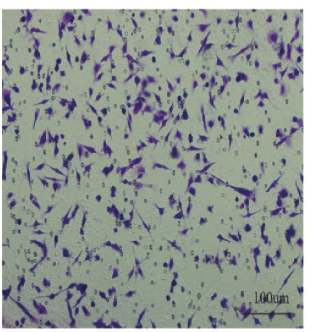

sh-MEG3-1

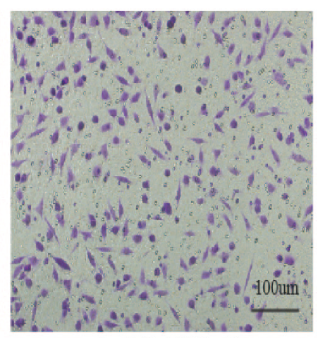

sh-MEG3-2

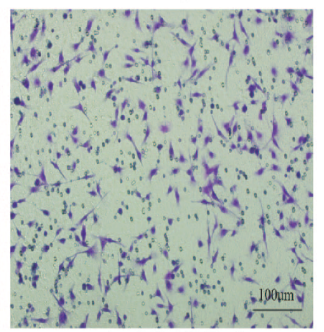

sh-MEG3-2

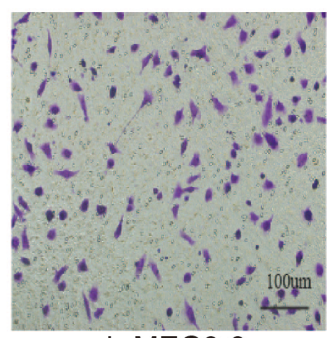

sh-MEG3-3

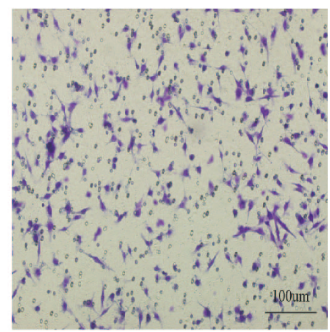

sh-MEG3-3
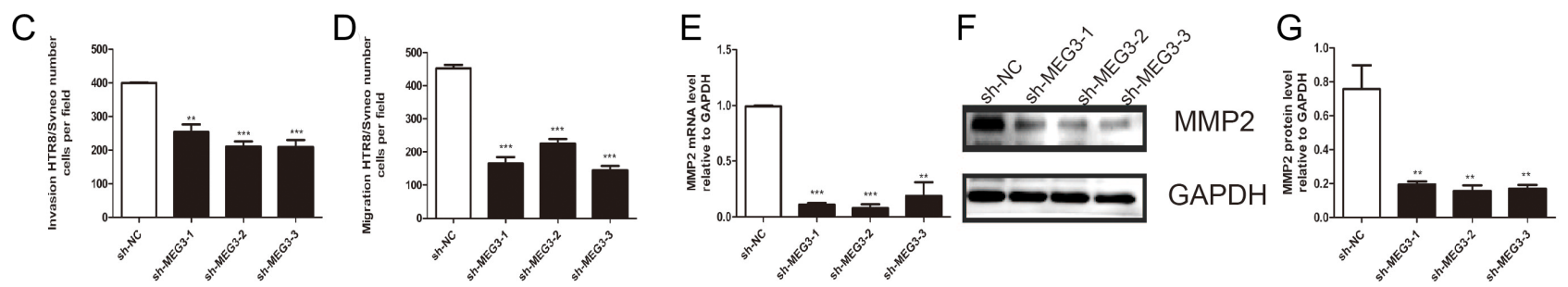

Figure 3 Knockdown of MEG3 decreased the migration and invasion of HTR8/SVneo cells. (A and C) Transwell assays showed that the migration of HTR8/SVneo cells with MEG silencing was significantly decreased compared to that of HTR8/SVneo cells transfected with sh-MEG-NC. (B and D) Matrigel invasion assay indicated the reduced invasion capabilities of MEG3 knockdown HTR8/SVneo cells compared with those transfected with sh-MEG3-NC. (E, F, and G) qRT-PCR and Western blotting were used to measure the mRNA and protein expression levels of MMP2 $\left(* * P<0.01,{ }^{* * *} P<0.001\right)$. 
a protein marker related to migration. We found that the mRNA and protein expression levels of MMP2 were both decreased in the sh-MEG3 group compared to the sh-MEG3-NC group (sh-MEG3 vs sh-MEG3-1, sh-MEG3-2, sh-MEG3-3: mRNA: $0.9933 \pm 0.006667$ vs $0.1087 \pm 0.01398,0.07867 \pm 0.03283,0.1853 \pm 0.1269$; protein: $\quad 0.7580 \pm 0.1397 \quad$ vs $0.1950 \pm 0.01790$, $0.1565 \pm 0.03320,0.1695 \pm 0.02281)($ Fig. 3E, F and G).

\section{Overexpression of MEG3 promoted HTR8/SVneo cell migration and invasion, which were then suppressed by the Notch1 inhibitor}

To further explore the involvement of the Notch1 pathway in the effect of MEG3 on cell migration and invasion, gain- and loss-of-function studies were conducted. In the transwell assay, ectopic expression of MEG3 promoted HTR8/SVneo cell migration capabilities compared to those of cells transfected with empty vector (NC group) (NC vs MEG3: $257.3 \pm 33.98$ vs $406.3 \pm 11.45$ ) (Fig. 4A and $\mathrm{C}$ ). The matrigel assay showed the same result ( $\mathrm{NC}$ vs MEG3: $191.7 \pm 6.064$ vs $368.7 \pm 1.667$ ) (Fig. $4 \mathrm{~B}$ and D). In addition, the restoration of Notch1 expression in HTR8/SVneo cells prevented the increase in their migration and invasion capabilities, which were induced by overexpression of MEG3 (Fig. 4A, B, C and D). We also detected the mRNA and protein expression levels of
Notch1 and MMP2. Overexpression of MEG3 increased Notch1 and MMP2 expression at both the mRNA and protein levels, and the addition of sh-Notch1 (a Notch1 inhibitor) reversed this result (Fig. 4E, F and G). Thus, these results indicated that MEG3 could promote HTR8/ SVneo cell migration and invasion capabilities, at least in part, by suppressing Notch1 signaling.

\section{Effects of MEG3 on HTR8/SVneo cell apoptosis and expression of $\mathrm{BCl}-2$ and $B A X$}

To further investigate the role of MEG3 in HTR8/ SVneo cell apoptosis, we performed a flow cytometryannexin V/PI stain assay in trophoblasts. When MEG3 was upregulated in HTR8/SVneo cells, the apoptotic cells were decreased significantly compared to those in the NC group (NC vs MEG3: 44.69 0.1790 vs $8.925 \pm 0.6899$ ) (Fig. $5 \mathrm{~A}$ and B). The sh-MEG3 group showed an increase in apoptotic cells (sh-MEG3-NC vs sh-MEG3-1, sh-MEG3-2, sh-MEG3-3: 4.395 \pm 0.5225 vs $21.17 \pm 0.4821,21.29 \pm 2.471,24.31 \pm 0.4446)$ (Fig. 5C and D). We further detected the mRNA and protein expression levels of Bcl-2 and BAX, two apoptosis-related markers. The results are as follows: overexpression of MEG3 in HTR8/SVneo cells, increased mRNA $(0.9900 \pm 0.005773$ vs 2.218 \pm 0.1932$)$ and protein $(0.5945 \pm 0.2341$ vs $2.611 \pm 0.3372)$ expression levels
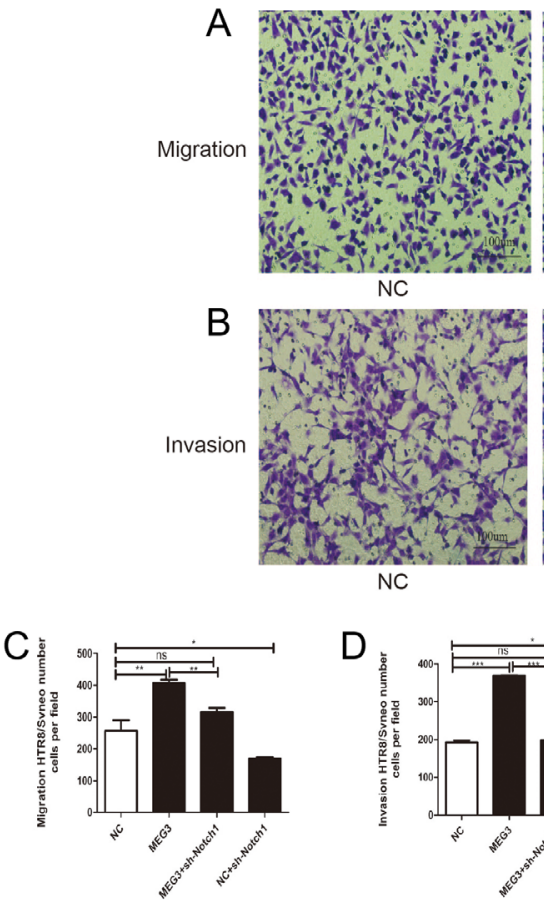

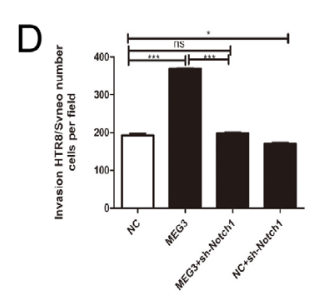

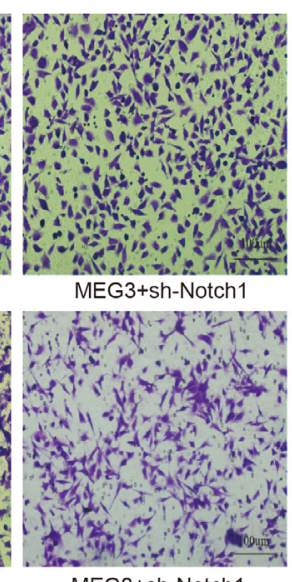

MEG3+sh-Notch1
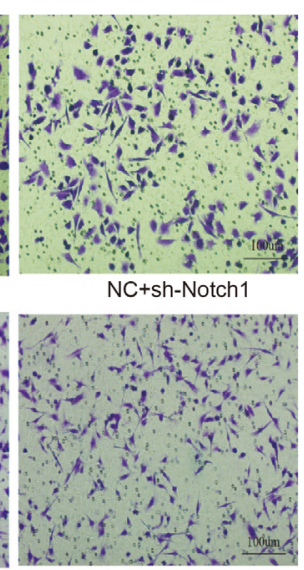

NC+sh-Notch1

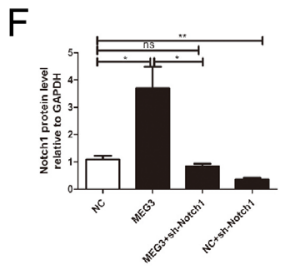

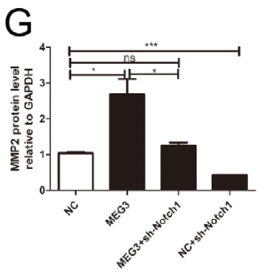

Figure 4 Overexpression of MEG3 promoted the migration and invasion of HTR8/SVneo cells in vitro. (A and C) Transwell assays showed that the migration of HTR8/SVneo cells MEG3 overexpression was significantly increased compared to that of cells transfected with NC (empty vector). Transfection of the sh-Notch1 vector rescued the migration of MEG3-overexpression cells. (B and D) Matrigel invasion assays indicated that MEG3 overexpression promoted invasion in HTR8/SVneo cells compared with the NC group. Moreover, transfection of the sh-Notch1 vector restored the invasion of MEG3-overexpression cells. (E, F, and G) qRT-PCR and Western blotting were used to measure the mRNA and protein expression levels of MMP2 and Notch1. ( $\left.{ }^{*} P<0.05, * * P<0.01, * * * P<0.001\right)$. 
A

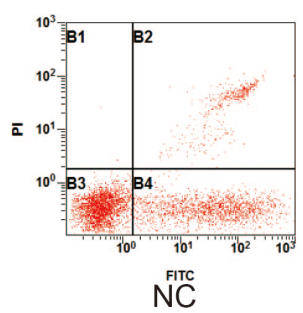

C

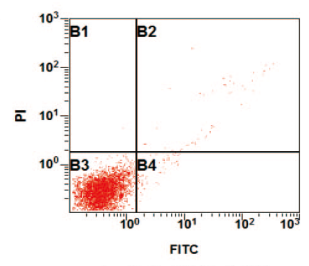

sh-MEG3-NC
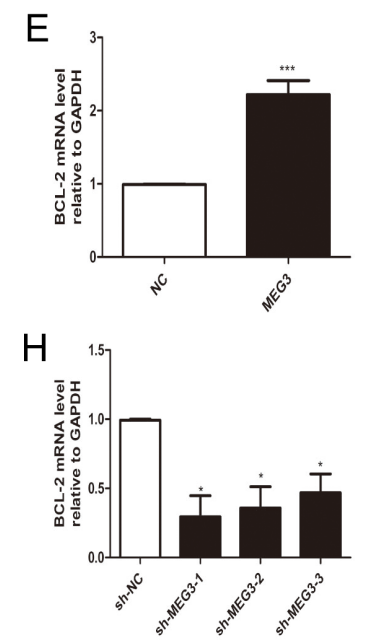
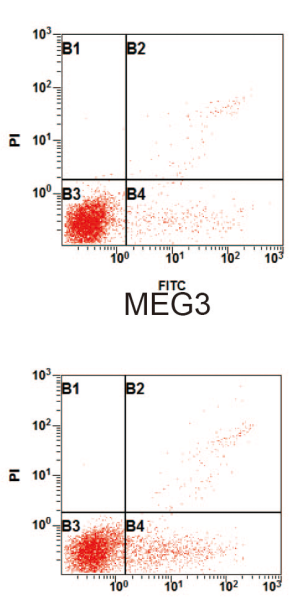

sh-MEGG3-1

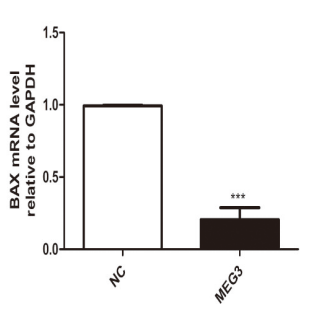

$\mathrm{F}$

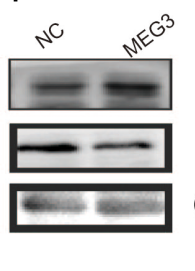

$\mathrm{Bcl}-2$

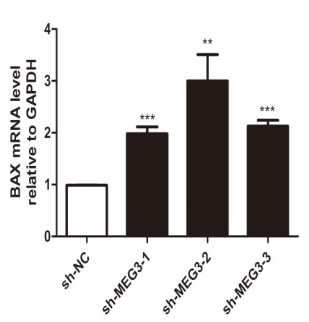

I
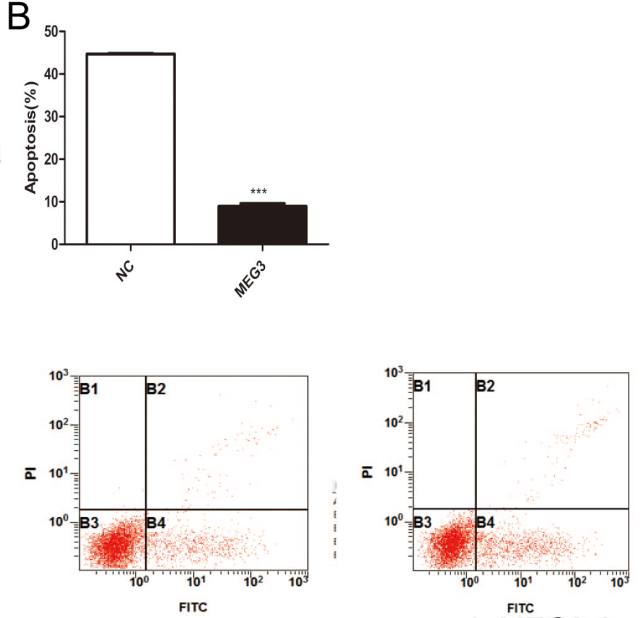

sh-MEGS-3
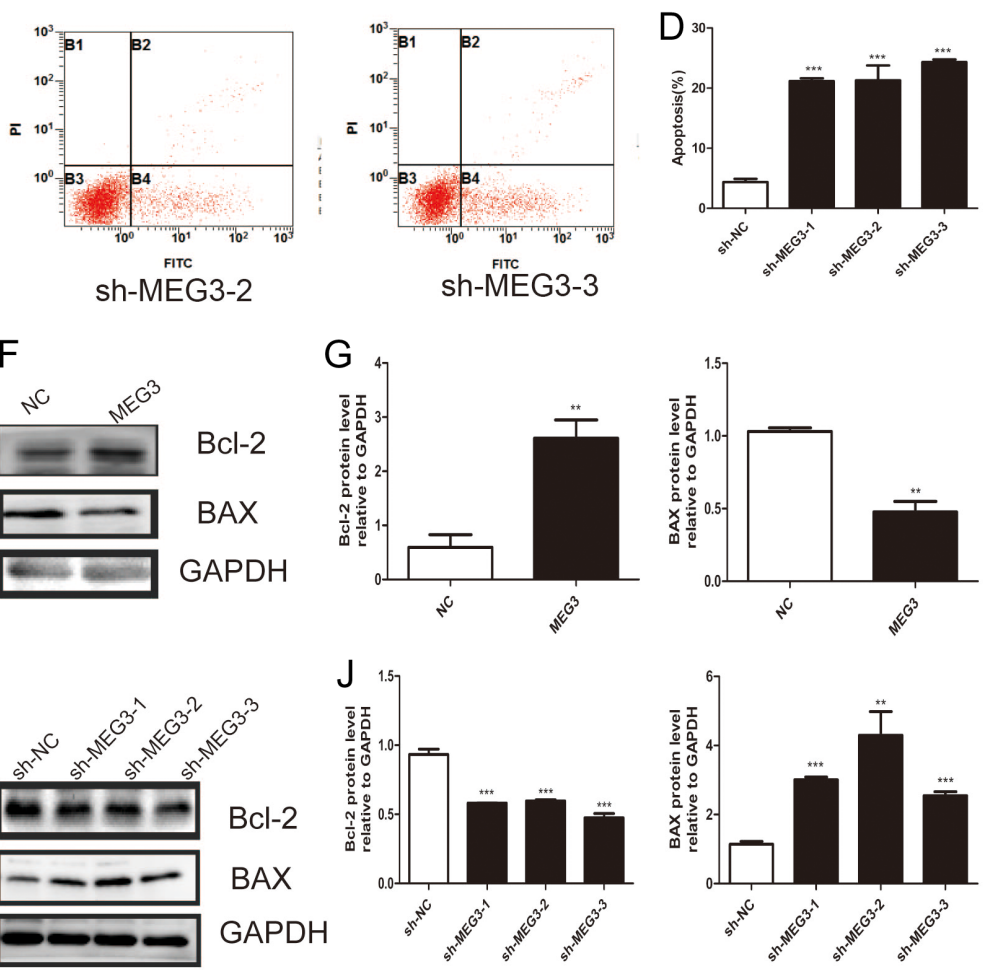

Figure 5 Overexpression and downregulation of MEG3 influenced HTR8/SVneo cell apoptosis in vitro. (A, B, C, and D) Flow cytometry assay indicated that the overexpression of MEG3 in HTR8/SVneo cells decreased cells apoptosis compared with cells transfected with the NC vector. HTR8/SVneo cells transfected with sh-MEG3 promoted cell apoptosis. (E, F, G, H, I, and J) qRT-PCR and Western blotting were used to measure the mRNA and protein expression levels of BCl-2 and BAX. $(* P<0.05, * * P<0.01, * * * P<0.001)$.

of Bcl-2, and decreased mRNA (0.9925 \pm 0.004787 vs $0.2061 \pm 0.08228)$ and protein $(1.030 \pm 0.02550$ vs $0.4770 \pm 0.07176$ ) expression levels of BAX (Fig. 5E, F and $\mathrm{G})$. The sh-MEG3 group showed the opposite results (Fig. $5 \mathrm{H}, \mathrm{I}$ and $\mathrm{J}$ ).

\section{Discussion}

Multiple studies have demonstrated that the expression levels of IncRNAs change extensively in preeclampsia (Song et al. 2017), especially those of MEG3, which was reported to be decreased by $80 \%$ in preeclampsia placenta compared to the controls. Previous studies have demonstrated that MEG3 could promote spiral artery remodeling by regulating vascular smooth muscle cell (VSMC) migration and apoptosis (Liu et al. 2017b, 2019). This finding is based on the regulation of motherderived cells. For cells of fetal origin, previous studies have indicated that overexpression of MEG3 could promote trophoblast cell migration capability, inhibit apoptosis, and decrease the protein levels of BAX. In contrast, downregulation of MEG3 elicited the opposite results (Zhang et al. 2015). However, the downstream target of MEG3 in the regulation of trophoblast cell migration is still largely unknown. Previous studies have verified that MEG3 could regulate cancer development and progression through the Notch signaling pathway (Zhaojian et al. 2012), and in rats with ischemic brain injury, MEG3 could regulate angiogenesis by activating Notch signaling (Liu et al. 2017a). Our previous studies have demonstrated that Notch1 is involved in the pathogenesis of preeclampsia (Qing-qing et al. 2015, Liu et al. 2016, Wang et al. 2019). Thus, we investigated whether the MEG3/Notch1 signaling pathway plays a role in the regulation of trophoblast cell biological function. 
In this study, we first examined the mRNA expression levels of MEG3 and Notch1 in preeclampsia placentas and normal controls. We found that the mRNA expression levels of MEG3 and Notch1 were decreased in preeclampsia placenta samples compared to the controls and that there was a positive correlation between them. This result strongly suggested that the MEG3/Notch1 signaling pathway was involved in the pathogenesis of preeclampsia. It would be more convincing if the placenta at the early stage of normal and preeclamptic pregnancies could be collected to know the expression levels of MEG3, which was one limitation of our present study. Furthermore, it is known that the process of placenta formation is very complicated and that the expression of the molecules involved may change dynamically with gestational age. Indeed, if we can collect enough placenta samples at different gestational ages to determine the dynamic expression of MEG3 and Notch1, it would strengthen our conclusions. However, due to clinical practice and ethical issues, we did not have access to enough placenta specimens.

We further investigated the relationship between MEG3 and Notch1 in the present study. The PCR and Western blot results demonstrated that upregulation of MEG3 promoted the mRNA and protein expression levels of Notch1, while downregulation of MEG3 suppressed the expression of Notch1. Then, to explore the role of MEG3 in trophoblast cell migration and invasion, MEG3 overexpression and silencing plasmids were transfected into HTR8/SVneo cells. We found that the ectopic expression of MEG3 promoted the migration and invasion of HTR8/SVneo cells. Conversely, knockdown of MEG3 suppressed the migration and invasion of HTR8/SVneo cells in vitro. Furthermore, gain- and loss-of-function studies were conducted to test whether MEG3 affected the bioactivity of HTR8/ SVneo cells by regulating Notch1 expression. The results revealed that overexpression of MEG3 in HTR8/SVneo cells increased the mRNA and protein expression levels of Notch1. Moreover, the knockdown of MEG3 elicited the opposite result. Importantly, by cotransfection of the MEG3 overexpression plasmid and Notch1 silencing plasmid into HTR8/SVneo cells, we found that the protein expression level of Notch1 returned to the level of the control group. Migration and invasion assays showed that restoration of Notch1 expression prevented MEG3medicated changes in these biological processes in HTR8/SVneo cells. Previous studies have demonstrated that MMP2 plays a key role in trophoblast migration and invasion. MMPs can be regulated at mutiple levels, including transcription, translation, secretion, and degradation (Sternlicht \& Werb 2001, Seval et al. 2004, Dimo et al. 2012, Wang et al. 2019). MEG3 could work as a transcriptional regulator, recruiting component of the polycomb repressor complex 2 on the genomic target site (Mondal et al. 2015). Furthermore, due to the formation of sequence-specific DNA/RNA triplex structures, MEG3 could also guide chromatin remodeling complexes to their action site on the genome (Mondal et al. 2015). In the cardiac fibroblasts, MEG3 could act as a chromatin-associated IncRNA and regulate the transcriptional activity of P53 on the promoter of MMP2 by direct interaction. Besides, inhibition of MEG3 expression leads to decreased expression and activity of MMP2, which could be cardioprotective (Piccoli et al. 2017). In our study, we speculated that MEG3 could also regulate trophoblast cell migration by regulating MMP2. Therefore, we also examined the mRNA and protein expression levels of MMP2, and the results were consistent with our functional assay. In summary, our study indicated that MEG3/Notch1 plays a positive role in placentation based on molecular and functional analyses.

Furthermore, mounting evidence has demonstrated that MEG3 has anti-apoptotic effects via the Bcl-2/Bax signaling pathway (Gu et al. 2017, Tong et al. 2019). Similarly, in our study, through flow cytometry, we found that overexpression of MEG3 decreased HTR8/SVneo cell apoptosis. Upregulation of MEG3 in HTR8/SVneo cells promotes the expression of $\mathrm{Bcl}-2$ at both the mRNA and protein levels and decreases the mRNA and protein levels of BAX. The downregulation of MEG3 elicited the opposite results. Previous studies have shown that Notch1 gene silencing in HTR8/SVneo cells could promote trophoblast cells apoptosis through regulation of the Bcl-2 protein family (Qing-qing et al. 2015). These results suggest that the MEG3/Notch1 signaling pathway plays a negative role in trophoblast apoptosis, which is vital for spiral artery remodeling.

More research is needed to explore the effects of the MEG3/Notch1 signaling pathway on trophoblast function. Trophoblasts, as epithelial cells, need to undergo a series of phenotypic changes, such as EMT, to invade the uterine wall of the mother (Floridon et al. 2000, Kokkinos et al. 2010, Wehrum et al. 2011). Previous studies have indicated that MEG3 plays a key role in the regulation of the EMT process, such as in lung cancer cells and gastric carcinoma cells (Terashima et al. 2017, Jiao \& Zhang 2019). Notch1 signaling is also involved in cell invasion by regulating the EMT process (Timmerman et al. 2004, Chen et al. 2018). We speculated that the MEG3/Notch1 signaling pathway modulates trophoblast cell function via the EMT process.

Moreover, research on the factors upstream of MEG3 may improve our understanding of the role of MEG3 in preeclampsia. Studies conducted by Yan et al. demonstrated that MEG3 could be a downstream target of miR-210 and that overexpression of miR-210 suppressed the expression levels of MEG3 (Yan et al. 2016). Our previous study indicated that miR-210 expression levels were elevated in preeclampsia and that upregulated miR-210 could attenuate the expression levels of Notch1 in HTR8/SVneo cells (Wang et al. 2019). Then we can infer that, in preeclampsia, overexpression of 
miR-210 decreased the expression levels of MEG3 and downregulation of MEG3 attenuated Notch1 expression. Downregulation of Notch1 inhibits the EMT process in trophoblasts, which is averse to spiral artery remodeling.

In conclusion, IncRNA MEG3 was demonstrated to be decreased in preeclampsia placenta, and downregulation of MEG3 may function as a suppressor in spiral artery remodeling by inhibiting the Notch1 signaling pathway. Furthermore, experiments carried out in vitro indicated a promising application of MEG3 overexpression to promote trophoblast migration and decrease apoptosis. Due to the complicated pathogenesis of preeclampsia, more in-depth studies are needed to explore the mechanism of Notch1 regulation under MEG3 dysfunction.

\section{Declaration of interest}

The authors declare that there is no conflict of interest that could be perceived as prejudicing the impartiality of the research reported.

\section{Funding}

This work was supported by the National Nature Science Foundation of China (No. 81741002 to Li Zou and No. 81703242 to Qingqing Luo).

\section{Author contribution statement}

Li Zou conceived this study. Rongli Wang performed experiments, analysed data, and wrote the paper.

\section{Acknowledgements}

The authors would like to thank all of the patients who participated in our study and the colleagues in the Department of Obstetrics and Gynecology for sample collection.

\section{References}

American College of Obstetricians and Gynecologists \& Task Force on Hypertension in Pregnancy 2013 Hypertension in pregnancy. Report of the American College of Obstetricians and Gynecologists' Task Force on Hypertension in Pregnancy. Obstetrics and Gynecology 122 1122-1131. (https://doi.org/10.1097/01.AOG.0000437382.03963.88)

Balik V, Srovnal J, Sulla I, Kalita O, Foltanova T, Vaverka M, Hrabalek L \& Hajduch M 2013 MEG3: a novel long noncoding potentially tumoursuppressing RNA in meningiomas. Journal of Neuro-Oncology 112 1-8. (https://doi.org/10.1007/s11060-012-1038-6)

Brosens M \& Renaer I 1972 On the pathogenesis of placental infarcts in pre-eclampsia. Journal of Obstetrics and Gynaecology 19794-799.

Chen DW, Wang H, Bao YF \& Xie K 2018 Notch signaling molecule is involved in the invasion of MiaPaCa2 cells induced by $\mathrm{CoCl} 2$ via regulating epithelial-mesenchymal transition. Molecular Medicine Reports 17 4965-4972. (https://doi.org/10.3892/mmr.2018.8502)

De Groot CJM, O'Brien TJ \& Taylor RN 1996 Biochemical evidence of impaired trophoblastic invasion of decidual stroma in women destined to have preeclampsia. American Journal of Obstetrics and Gynecology 175 24-29. (https://doi.org/10.1016/s0002-9378(96)70245-4)
Dimo B, loannidis I, Karameris A, Vilaras G, Tzoumakari P, Nonni A, Patsouris E \& Lazaris AC 2012 Comparative study of the immunohistochemical expression of tissue inhibitors of metalloproteinases 1 and 2 between clearly invasive carcinomas and 'in situ' trophoblast invasion. Medical Oncology 29 2270-2275.

Floridon C, Nielsen O, Holund B, Sunde L, Westergaard JG, Thomsen SG \& Teisner B 2000 Localization of E-cadherin in villous, extravillous and vascular trophoblasts during intrauterine, ectopic and molar pregnancy. Molecular Human Reproduction 6 943-950. (https://doi.org/10.1093/ molehr/6.10.943)

Gu L, Zhang J, Shi M, Zhan Q, Shen Baiyong \& Peng C 2017 LncRNA MEG3 had anti-cancer effects to suppress pancreatic cancer activity. Biomedicine and Pharmacotherapy 89 1269-1276. (https://doi. org/10.1016/j.biopha.2017.02.041)

Guo Q, Qian Z, Yan D, Li L \& Huang L 2016 LncRNA-MEG3 inhibits cell proliferation of endometrial carcinoma by repressing Notch signaling. Biomedicine and Pharmacotherapy 82 589-594. (https://doi. org/10.1016/j.biopha.2016.02.049)

Jiao J \& Zhang S 2019 Long non-coding RNA MEG 3 suppresses gastric carcinoma cell growth, invasion and migration via EMT regulation. Molecular Medicine Reports 20 2685-2693. (https://doi.org/10.3892/ mmr.2019.10515)

Kokkinos MI, Murthi P, Wafai R, Thompson EW \& Newgreen DF 2010 Cadherins in the human placenta-epithelial-mesenchymal transition (EMT) and placental development. Placenta 31 747-755. (https://doi. org/10.1016/j.placenta.2010.06.017)

Limbourg FP, Takeshita K, Radtke F, Bronson RT, Chin MT \& Liao JK 2005 Essential role of endothelial Notch1 in angiogenesis. Circulation 111 1826-1832. (https://doi.org/10.1161/01.CIR.0000160870.93058.DD)

Liu X, Luo Q, Zheng Y, Liu X, Hu Y, Liu W, Luo M, Tao H, Wu D, Zhao Y et al. 2016 Notch1 impairs endothelial progenitor cell bioactivity in preeclampsia. Reproductive Sciences 24 47-56.

Liu J, Li Q, Zhang K-S, Hu B, Niu X, Zhou S-M, Li S-G, Luo Y-P, Wang Y \& Deng ZF 2017a Downregulation of the long non-coding RNA Meg3 promotes angiogenesis after ischemic brain injury by activating Notch signaling. Molecular Neurobiology 54 8179-8190. (https://doi. org/10.1007/s12035-016-0270-z)

Liu W, Liu X, Luo M, Liu X, Luo Q, Tao H, Wu D, Lu S, Jin J, Zhao Y et al. $2017 b$ DNK derived IFN- $\gamma$ mediates VSMC migration and apoptosis via the induction of LncRNA MEG3: a role in uterovascular transformation. Placenta 50 32-39. (https://doi.org/10.1016/j.placenta.2016.12.023)

Liu W, Luo M, Zou L, Liu X, Wang R, Tao H, Wu D, Zhang W, Luo Q \& Zhao Y 2019 UNK cell-derived TGF-B1 regulates the long noncoding RNAMEG3 to control vascular smooth muscle cell migration and apoptosis in spiral artery remodeling. Journal of Cellular Biochemistry 120 15997-16007. (https://doi.org/10.1002/jcb.28878)

Meekins JW, Pijnenborg R, Hanssens M, McFadyen IR \& van Asshe A 1994 A study of placental bed spiral arteries and trophoblast invasion in normal and severe pre-eclamptic pregnancies. British Journal of Obstetrics and Gynaecology 101 669-674. (https://doi.org/10.1111/j.1471-0528.1994. tb13182.x)

Mondal T, Subhash S, Vaid R, Enroth S, Uday S, Reinius B, Mitra S, Mohammed A, James AR, Hoberg E et al. 2015 MEG3 long noncoding RNA regulates the TGF-b pathway genes through formation of RNA DNA triplex structures. Nature Communications 6 7743. (https://doi. org/10.1038/ncomms8743)

Myatt L 2002 Role of placenta in preeclampsia. Endocrine 19 103-111. (https://doi.org/10.1385/ENDO:19:1:103)

Piccoli M, Gupta SK, Viereck J, Foinquinos A, Samolovac S, Kramer FL, Garg A, Remke J, Zimmer K, Batkai S et al. 2017 Inhibition of the cardiac fibroblast-enriched IncRNAMeg3 prevents cardiac fibrosis and diastolic dysfunction. Circulation Research 121 575-583. (https://doi. org/10.1161/CIRCRESAHA.117.310624)

Qing-qing L, Liu X, Zheng Y, Zhao Y \& Zou L 2015 Effect of Notch1 gene silence on proliferation and apoptosis of trophoblast cells. Maternal and Child Health Care in China 30 755-757.

Seval Y, Ramazan GKA, Demir R \& Asar M 2004 Distribution patterns of matrix metalloproteinase (MMP)-2 and -9 and their inhibitors (TIMP-1 and TIMP-2) in the human decidua during early pregnancy. Acta Histochemica 106 353-362. (https://doi.org/10.1016/j.acthis.2004.07.005)

Song X, Luo X, Gao Q, Wang Y, Gao Q \& Long W 2017 Dysregulation of LncRNAs in placenta and pathogenesis of preeclampsia. Current Drug 
Targets 18 1165-1170. (https://doi.org/10.2174/138945011866617040 4160000)

Sternlicht MD \& Werb Z 2001 How matrix metalloproteinases regulate cell behavior. Annual Review of Cell and Developmental Biology 17 463-516. (https://doi.org/10.1146/annurev.cellbio.17.1.463)

Sun L, Li Y \& Yang B 2016 Downregulated long non-coding RNA MEG3 in breast cancer regulates proliferation, migration and invasion by depending on P53's transcriptional activity. Biochemical and Biophysical Research Communications 478 323-329. (https://doi.org/10.1016/j. bbrc.2016.05.031)

Terashima M, Tange S, Ishimura A \& Suzuki T 2017 MEG3 long noncoding RNA contributes to the epigenetic regulation of epithelial-mesenchymal transition in lung cancer cell lines. Journal of Biological Chemistry 292 82-99. (https://doi.org/10.1074/jbc.M116.750950)

Timmerman LA, Grego-Bessa J, Raya A, Bertrán E, Pérez-Pomares JM, Díez J, Aranda S, Palomo S, McCormick F, Izpisúa-Belmonte JC et al. 2004 Notch promotes epithelial-mesenchymal transition during cardiac development and oncogenic transformation. Genes and Development 18 99-115. (https://doi.org/10.1101/gad.276304)

Tong P, Peng QH, Gu LM, Xie WW \& Li WJ 2019 LncRNA-MEG3 alleviates high glucose induced inflammation and apoptosis of retina epithelial cells via regulating miR-34a/SIRT1 axis. Experimental and Molecular Pathology 107 102-109. (https://doi.org/10.1016/j.yexmp.2018.12.003)

Wang C, Yan G, Zhang Y, Jia X \& Bu P 2015 Long non-coding RNA MEG3 suppresses migration and invasion of thyroid carcinoma by targeting of Rac1. Neoplasma 62 541-549. (https://doi.org/10.4149/neo_2015_065)

Wang R, Liu W, Liu X, Liu X, Tao H, Wu D, Zhao Y \& Zou L 2019 MicroRNA-210 regulates human trophoblast cell line HTR-8/SVneo function by attenuating Notch1 expression: implications for the role of microRNA-210 in pre-eclampsia. Molecular Reproduction and Development 86 896-907. (https://doi.org/10.1002/mrd.23154)

Wehrum MJ, Buhimschi IA, Salafia C, Thung S, Bahtiyar MO, Werner EF, Campbell KH, Laky C, Sfakianaki AK, Zhao G et al. 2011 Accreta complicating complete placenta previa is characterized by reduced systemic levels of vascular endothelial growth factor and by epithelialto-mesenchymal transition of the invasive trophoblast. American Journal of Obstetrics and Gynecology 204 411.e1-411.e11. (https://doi. org/10.1016/j.ajog.2010.12.027)

Yan YY, Wang ZH, Zhao L, Song DD, Qi C, Liu LL \& Wang JN 2016 MicroRNA-210 plays a critical role in the angiogenic effect of isoprenaline on human umbilical vein endothelial cells via regulation of noncoding RNAs. Chinese Medical Journal 129 2676-2682. (https://doi. org/10.4103/0366-6999.193452)

Zhang X, Zhou Y, Mehta KR, Danila DC, Scolavino S, Johnson SR \& Klibanski A 2003 A pituitary-derived MEG3 isoform functions as a growth suppressor in tumor Cells. Journal of Clinical Endocrinology and Metabolism 88 5119-5126. (https://doi.org/10.1210/jc.2003-030222)

Zhang Y, Zou Y, Wang W, Zuo Q, Jiang Z, Sun M, De W \& Sun L 2015 Down-regulated long non-coding RNA MEG3 and its effect on promoting apoptosis and suppressing migration of trophoblast cells. Journal of Cellular Biochemistry 116 542-550. (https://doi.org/10.1002/jcb.25004)

ZhaoJian G, ShanShan Z, Zhang WL, Huang HB, Qiao LI, Hao D, Ma J, Xiang ZM, Xiong W, XiaoLing LI et al. 2012 Long non-coding RNAs in cancer. Science in China 55 1120-1124.

Zhou Y, Zhang X \& Klibanski A 2012 MEG3 noncoding RNA: a tumor suppressor. Journal of Molecular Endocrinology 48 R45-R53. (https:// doi.org/10.1530/JME-12-0008)

Received 19 December 2019

First decision 21 February 2020

Revised manuscript received 28 March 2020

Accepted 9 April 2020 